\title{
TOWARDS DISCOURSE-ORIENTED NONMONOTONIC SYSTEM
}

\author{
Barbara Dunin-Kęplicz Witold Łukaszewicz \\ Institute of Informatics \\ Warsaw University \\ P.O.Box 1210 \\ 00-901. Warszawa, FOLAND
}

\begin{abstract}
The purpose of this paper is to analyse the phenomenon of nonmonotonicity in a natural language and to formulate a number of general principles which should be taken into consideration while constructing a discourse oriented nonmonotonic formalism.
\end{abstract}

\section{INTRODUCTION}

For the purpose of this paper we assume that to understand a discourse is to specify all the conclusions derivable from the discourse itself knowledge about the world and knowledge of language use.

To complete discourse-connected conclusions, a number of various linguistic phenomena must be resolved. The solution of such problems as anaphora, quantification, negation and so on, should be regarded as full right conclusions. The reason is that this purely linguistic information is necessary for obtaining the essential one, describing the external world under consideration.

The measure of quality of any discourse analysis system is the adequacy of its inference capabilities to those of human language users. This implies that any such a high quality system must provide some mechanism modelling common sense reasoning.

In everyday life we are continously forced to accept various conclusions which we are prepared to reject when our knowledge increases. The ability of drawing such cancellable inferences, beliefs in AI terminology, makes common sense reasoning nonmonotonic in the sense that the set of derivable conclusions does not increase monotonically with the set of premises, as in standard logics.

Earlier experiences have proved that ad hoc nonmonotonic tools were ineffective. They have seemed to work for the simplest cases only. Since they lacked theoretical foundations, their behaviour has been unclear in more complex situations.

Recently there have been a number of attempts to formalize various nonmonotonic mechanisms (see (AI, 1980), (AAAI, 1984)).

In this paper we analyse the phenomenon of nonmonotonicity in a natural language. We also formulate a number of general principles which should be taken into account while specifying a discourse-oriented nonmonotonic formalism.

It is well recognized that ordering of dis. course utterances is essential for its understanding. On the other hand, logical systems lack mechanisms capturing this property. It follows, therefore, that to model the dynamic nature of a discourse, some kind of structuralization is needed. A very natural, both computationally and conceptually, discourse structuralization has been suggested by Kamp (Kamp, 1981).
According to Kamp, a discourse is represented as a D (iscourse) R (epresentation) S (tructure). Reughly speaking, DRS is a sequence of $D$ (iscourse) $R$ (epresentations).A DR is nothing else than a partial model, describing discourse objects and their relations. A DRS is constructed as follows. We start with the empty DR. Each discourse utterance extends the actual DR by adding appropriate information contained in the utterance. To construct any DR, some kind of reasoning mechanism is needed. We postulate the application of the nonmonotonic inference system for that purpose.

\section{NONMIONOTONICITY IN DISCOURSE UNDERSTANDING}

To show the universality of nonmonotonicity in a natural language, we shall present a number of examples concerning various linguistic phenomena commonty occurring in a discourse.

One should be aware that analogous treatment of such different concepts as, for instance, anaphora and presupposition is inappropriate. Anaphora, as well as quantification or negation are purely surface phenomena. One must resolve -them while processing a discourse, but their solutions lead to conclusions describing the discourse rather, than the external world under consideration. For this reason we shall refer to those conclusions as surface conclusions. Presupposition, on the other hand, as well as time line construction, conditionals or various conversational rules, concerns conclusions describing directly the world being represented by a discourse. We shall refer to those conclusions as general conclusions.

Our purpose is not to review all the linguistic phenomena dealing with nonmonotonicity, but to demonstrate that

(i) both kinds of conclusions are subject to invalidation,

(ii) cancellable conclusions of both types can be supported either by semantic or pragmatic sources.

This leads to an important observation that nonmonotonicity exists on various levels of discourse understanding, viewing both processing method and data to be processed.

\section{Surface conclusions}

The following examples of pronoun anaphora and quantification show that semanticmbased as well as progmatic-based surface conclusions can be invalidated.

Example 1 (pronoun anaphora)

(1) The Vice-President entered the President's office. He was nervous and clutching his briefcase. 
This example is from (Ascher, 1984). There are semantic reasons supporting the conclusion that the Vice-President is the one who is nervous given the information that he has a meeting with the president. This conclusion can be, however, overturned by adding

(2) After all, he couldn't fire the Vice-President without making trouble for himself with the chairman of the board.

Example 2 (pronoun anaphora)

(3) Peter was sitting in a room. When John entered the room he seemed nervous.

Although there are two possible referents of "he" in (3), it is pragmatically well motivated that "he" refers to "Peter". This follows from Grices Cooperative Principle. Assuming that the speaker is obeying it, he should replace (5) by the unmistakable

(4) Peter was sitting in a room. Entering the room, John seemed nervous.

if it were the case that John had been nervous. Nevertheless, this preferred coreference is invalidated if (1) is extended to

(5) Peter was sitting in a room. When John entered the room he seemed nervous. He was afraid of Peter.

Example 3 (quantification)

(6) There are three men in a room. Every man loves a woman.

There are clearly two possible paraphrases of (6):

(i) There are three men in a room. There is a woman such that she is loved by each of them.

(8) There are three men in a room. For each man there is a woman such that he loves her.

Applying his "ordering principles", based on the observation that humans are used to handle information from left to right, Hintikka in (Hintikka, 1977) coxvincingly suggests that (8) is pragmatically preferred reading for (6). Nevertheless, this conclusion may be overturned. For instance, if $(6)$ is extended to

(9) There are three men in a room. Every man loves a woman. Her name is Melinda.

Example 4 (quantification)

(10) A rifle has been given to all soldiers.

From among two possible readirgs of (10), the one asserting that different rifles have been given to different soldiers should be semantically preferred. But this interpretation is immediately invalidated when (10) is replaced by

(11) A rifle has been given to all soldiers. It has turned out to be broken.

\section{General conclusions}

The following examples of presupposition illustrate that semantic-based as well as pragmatic-based general conclusions are subject to cancellation.

Example 5 (presupposition)

(1.2) John regrets that he didn't win the prize.
(12) presupposes that John did not win the prize. Nevertheless, this general conclusion is immediately invalidated when (12) is extended to ed to

(1.3) John regrets that he didn't win the prize. In fact, he doesn't know that he did.

Example 6 (presupposition)

(14) "If I had money I would buy a rifle to defend you from the Wolf", Red Riding Hood said to her Grandmother.

(14) presupposes that RRI had no money. This follows from the pragmatic rules of interpretation of counterfactuals in Inglish. The cancelo ability of this conclusion is easily seen, if (14) is extended to

(15) "If I had money I would buy a rifle to defend you from the Wolf", RRH said to her Grandmother. In fact, RRI was cheating her. She had money, but she wanted to buy herself a new dress.

According to the presented examples, nonmonotonicity is not a local phenomenon. It exists on various levels of discourse analysis. It seems, therefore, that no high quality discourse understanding system can ignore this fact.

\section{TOWARDS A DISCOURSEMORIENTED} NONMONOTONIC FORMALISM

Although a great number of various nonmonotonic ( $\mathrm{nm}$, for short) formalisms can be found in Al literature, none of them seems to be fully appropriate for the purpose of discourse understanding. In our opinion, only default logic partially. captures the expressive power of natural language. Deriving from Minsky's frame concept, this formalism has turred out to be useful for various natural language applications (see (Dunin. -Kęplicz, 1984), (Mercer, Reiter, 1984).

Default logic has been introduced by Reiter in (Reiter, 1980) to model default reasoning, i.e., the drawing of plausible conclusions from incomplete informalion in the absence of evidence to the contrary. A typical example of default reasoning is the inference rule "Typically birds fly". If Tweety is a bird, then in the absence of evidence to the contrary, we normally assume that Tweety flies. But we are prepared to reject this conclusion, if: we learn that Tweety is a pinguin,

In default logic the rule about birds is represented by the following default:

$$
\operatorname{bird}(x) \text { : M flies }(x) / \text { flies }(x)
$$

with the intended interpretation: "for each individual $x$, if $x$ is a bird and it is consistent to asm sume that $x$ flies, then it may be assumed that $x$ flies".

In default logic the world under consideration is represented as a default theory, i,e, a pair consisting of a set of first-order formulae, the axioms of the theory, and a set of defaults. Der faults extend the information contained in axioms by sanctioning plausible, but not necessarily true, conclusions.

A set of formulae derivable from a given default theory is called an extension of the theory and is interpreted as a set of beliefs about the world being madelled, (see (Reiter, 1980) for details). 
Specifying a $\mathrm{nm}$ discourse-oriented formalism is a difficult problem. The first step is to determine all its relevant properties. In this section we shall try to accomplish this point and to discuss some weaknesses of default logic in this respect.

The first problem is to choose an appropriate standard logic as a basis of the constructed system. Although the majority of existing $\mathrm{nm}$ formalisms are based on classical first-order logic, it is well recognized that to capture the structure of natural languages, at least intensional logic, preferable with tense operators, is required. It should be, however, stressed that for computational reasons some compromise between adequacy and simplicity is necessary.

The next step is to add a nonmonotonic deductive structure to the chosen monotonic logic. This amounts to the following task. Given a theory A, i.e., a set of formulae describing relevant information about a world, determine a set $\mathrm{E}(\mathrm{A})$ of conclusions nonmonotonically derivable from A. The general idea is to identify this set with the set of conclusions monotonically derivable from some extension of A. For this reason $\mathrm{E}$ (A) is called an extension of $A$, and is interpreted a.s the set of plausible conclusions about the world under consideration.

Defining a language and $\mathrm{nm}$ deductive structure, determines a $\mathrm{nm}$ system. But to make the system applicable for discourse processing, a number of additional factors should be taken into consideration.

First, a semantics must be specified. In particular, a model regarded as a $\mathrm{nm}$ description of a real world under consideration should be defined.

Second, a method of determining whether a conclusion can be inferred from a given set of premises should be specified. Because nm derivability depends not only on what can be proved, but also on what cannot be proved, there are technical problems involved here. In particular, even, if the system is based on semi-decidable first-order logic, its proof theory is generally undecidable. This means that some kind of heuristics is necessary, and the system will sometimes arrive at mistaken conclusions. Nevertheless, it is often sufficient to consider only models with finite domains limited to the individuals explicitely occurring in the discourse. In such a case, the logic we are dealing with is, in fact, the propositional one. The nm formalism based on propositional logic is, of course, decidable.

Third, beliefs revision, that is a method of reorganizing world model when new information leads to inconsistency should be specified. This very difficult problem has been marginally treated in the most of existing $\mathrm{nm}$ systems. On the other hand, since each discourse utterance modifies the actual world model, beliefs revision seems one of the central problems of discourse analysis.

Fourth, the existence of extensions should be guaranteed. Although this demand seems obvious, it is not satisfied in many existing $\mathrm{nm}$ formalisms, in particular, in Reiter's default logic.
An example of a discourse, whose representation in Reiter"s system lacks an extension, can be found in ( \pm ukaszewicz, 1984). This paper pret sents also an alternative formalization of default logic which satisfies the above postulated property.

Fifth, the system should adequately model common sense reasoning. In terms of extension, this means that such an extension should contain those discourse-connected conclusions which would be drawn by humans. In our opinion, none of existing $\mathrm{nm}$ formalisms fully captures this requirement. The detailed discussion of this hypothesis can be found in (Eukaszewicz, 1986).

In the majority of $\mathrm{nm}$ systems the notion of an extension is defined in a way admitting the existtence of many different extensions for a given theory. This poses the question of how to define the set of conclusions derivable from such a theory. There are two possibilities. First, to view each extension as an alternative set of beliefs one can hold about a world under consideration. In fact, this solution has been adopted in default logic. Second, to identify the set of conclusions with the intersection of all extensions. Both solutions are reasonable but have different interpretam tions. The first represents what an agent beliefs about a world, the second, what an outside observer would know about the agent's beliefs, given the set of the agent's premises about the world.

In our opinion, the second solution is better motivated in discourse processing. This follows from the fact that both the speaker and the hearer try to achieve a common representation of a discourse. This would be impossible, if each of them accepted a different extension.

\section{REFERENCES}

AAAI (1984) Non-Monotonic Reasoning Workshop, New Paltz, NY, October 17-19, 1984.

AI (1980) Artificial Intelligence. Vol, 13/1,2.

Ascher, N., (1984) Linguistic Understanding and Non-Monotonic Reasoning. In AAAl-84, pp. 1-20.

Dunin-Keeplicz B., (1984) Default Reasoning in Anaphora Resolution. Proc. 6th ECAI. pp. 157-166.

Hintikka J., (1977) Quantifiers in Natural Languages: Some Logical Problems II. Linguistics and Philosophy 1, pp. 153-172.

Kamp H., (1981) A Theory of Truth and Semantic Representation. Formal Methods in the Study of Language, Amsterdam, pp. 277-322.

Łukaszewicz.W., (1984) Considerations on Default Logic. In AAAI-84, pp. 165-193.

Łukaszewicz W., (1986) Formalization of Knowm ledge and Ignorance: An Introduction to Nonm monotonic Reasoning, CC AI, to appear.

Mercer R., Reiter, R., (1982) The representation of Presuppositions Using Defaults. B.C.University. Technical. Report 82-1.

Reiter R., (1980) A Logic for Default Reasoning. Artificial Intelligence 13/1,2, pp. 81-132. 